\title{
The feasibility of vasopressin administration via laryngeal mask airway using a porcine model
}

BY YU-YING LIAO, CHIEN-CHIN HSU, HOW-RAN GUO, KUO-TAI CHEN

\section{Abstract}

Background. In pre-hospital situations, delay in resuscitation might carry extra risks for patients, so resuscitative measures should be rapid, easyto use, and effective. A laryngeal mask airway (LMA) is a quickly placed supraglottic airway that may be used as a route for drug administration. Vasopressin is a vasopressor and might be absorbed well via the mucosa of the airways and alveoli. We conducted this animal study to verify the feasibility of administering vasopressin via a LMA.

Methods. Twenty-four Yorkshire pigs were anesthetized and randomly divided into four groups. The pigs in Groups Placebo and tracheal tube (TT) were intubated with a cuffed tracheal tube, and those in Groups LMA and laryngeal mask airway and a catheter (LMAC) underwent a size 4 LMA insertion. In the LMAC group, an aerosolized catheter was placed into the trachea through a LMA to deliver the drug. All pigs were able to breathe spontaneously without the assistance of a ventilator. The placebo group received $5 \mathrm{ml}$ of distilled water via a tracheal tube. The other 
groups received $1 \mathrm{U} / \mathrm{kg}$ vasopressin, which was diluted to a total volume of $5 \mathrm{ml}$ with distilled water via the varied routes. The heart rates and arterial pressures were recorded before and after drug administration.

Results. The mean arterial pressure (MAP) and diastolic arterial pressure (DAP) increased significantly and maintained a plateau from 3 to $7 \mathrm{~min}$ in Group TT and 2 to 29 min in Group LMAC. Group LMA and Group Placebo demonstrated only one occasional elevation in MAP and no changes in DAP. Furthermore, the heart rate decreased significantly from 2 to 29 min in Group LMAC.

Conclusions. In this porcine model, vasopressin administered via an aerosolized catheter and ventilated with a LMA demonstrated a positive and prolonged pressor effect. The results suggest that an aerosolized catheter placed through a LMA, may be a practical alternative route for vasopressin administration, and that the effective duration of vasopressin is long enough to cover the period of pre-hospital management.

Key words: laryngeal mask airway, vasopressin, airway, pre-hospital

\section{Introduction}

In pre-hospital situations, the feasibility of varied resuscitative management is crucial for patients and emergency medical technicians (EMTs). Out-of-hospital management should be rapid, easy-to use, and effective, even in rigorous situations. A prolonged stay at the scene might carry extra risks for patients. $(1,2)$

Intravenous, intraosseous, and administration via tracheal tube, are all practical methods for pre-hospital drug delivery. However, establishment of these routes is usually difficult and time-consuming in out-of-hospital situations. (3-5) These procedures prolong the length of stay and might be misplaced at the scene. $(3,5)$

A laryngeal mask airway (LMA) is an effective supraglottic airway device and is widely used by EMTs. A LMA can be placed quickly and easily by 
unfamiliar healthcare workers and the skill can be retained without frequent training. $(6,7)$ Furthermore, previous studies have revealed its potential to serve as a route for drug administration. (8-10)

Vasopressin is a potent vasopressor with extended purposes in the treatment of severe trauma, septic shock, cardiac diseases and during cardiopulmonary resuscitation. (11-22) In addition, a few researchers have found that vasopressin is absorbed well via the mucosa of the airway and alveoli. (23-25) The combination of a LMA and vasopressin could be a practical management tool in pre-hospital settings. Accordingly, we conducted this animal study to verify the feasibility of administering vasopressin via a LMA.

\section{Materials and methods}

The project was approved by the animal investigation committee of ChiMei Medical Center. The animals were managed in accordance with national and institutional guidelines. Sixteen female and 8 male Yorkshire pigs, with an average weight of $22.1 \mathrm{~kg}$ (range 16.5-25.7 kg; median $22.5 \mathrm{~kg}$ ), were used in this study (table 1). Before the experiment, the pigs were fasted overnight but given unrestricted access to water. The animals were premedicated with Tiletamine/Zolazepam $(2.5 \mathrm{mg} / \mathrm{kg})$ intramuscularly and were fixed in a dorsal recumbent position. Anesthesia was inducted with $10 \mathrm{mg} / \mathrm{kg}$ pentothal injected into an ear vein. All of the pigs were able to breathe spontaneously without ventilator assistance and none of the animals needed an additional anesthetic agent to maintain anesthesia during the experiment. A femoral artery was catheterized for continuous arterial pressure and heart rate monitoring, and $3000 \mathrm{U}$ intravenous heparin was administered to avoid intra-catheter clot formation. The pigs were then randomly divided into four groups, with each group comprising 6 animals. The animals in the Placebo and tracheal tube (TT) groups were intubated with a cuffed tracheal tube (internal diameter $7 \mathrm{~mm}$ ) and those in the LMA and laryngeal mask airway and a catheter (LMAC) groups underwent a size 4 LMA (LMA-Classic ${ }^{\text {TM}}$; The Laryngeal Mask 
Company, Henley on Thames, UK) insertion. In the LMAC group, a catheter (MADett ${ }^{\circledR}$; Wolfe Tory Medical, Salt Lake City, America) was placed into the trachea through the LMA to deliver the medication. The catheter was designed to aerosolize the injected solution, which facilitated the absorption of the drug. We used a fibrobronchoscopy to confirm the position of the tracheal tube, ensure that the opening of the larynx was faced directly towards the lower aperture of the LMA, and verify that the tip of the MADett ${ }^{\circledR}$ catheter was located in the trachea.

After completing the above procedures, we allowed a 20-minute stabilization period to achieve a steady state.

Group Placebo: $5 \mathrm{ml}$ of distilled water was administered via the tracheal tube.

Group TT: $1 \mathrm{U} / \mathrm{Kg}$ of vasopressin, diluted to a total volume of $5 \mathrm{ml}$ with distilled water, was administered via the tracheal tube.

Group LMA: $1 \mathrm{U} / \mathrm{Kg}$ of vasopressin, diluted to a total volume of $5 \mathrm{ml}$ with distilled water, was administered via the upper end of the LMA.

Group LMAC: $1 \mathrm{U} / \mathrm{Kg}$ of vasopressin, diluted to a total volume of $5 \mathrm{ml}$ with distilled water, was administered via the MADett ${ }^{\circledR}$ catheter.

Five forceful artificial ventilations were given via tracheal tube or LMA to help the spraying of medication after various drugs were administered. The heart rate and arterial pressure were recorded before administration, at 30 seconds after drug administration, every minute for 9 minutes, and then every 2 minutes for 29 minutes. At the end of the experiment, the animals were killed using an injection of potassium chloride solution 10 mEq.

The results are presented as medians, unless otherwise stated. Differences in heart rate and arterial pressure between the four groups were compared using the Kruskal-Wallis test, followed by the MannWhitney U test. Using the data before drug administration as the baseline, changes in hemodynamic variables were investigated using the 
Friedman test followed by the Wilcoxon signed rank test. All statistical tests were performed using SPSS software package (SPSS 12.0), with a two-tailed significance level of 0.05 .

\section{Results}

After drugs were administered via varied routes, the mean arterial pressure (MAP) and diastolic arterial pressure (DAP) of Group LMAC decreased significantly at 0.5 minutes and returned to baseline soon after, at 1 minute postinjection. Both MAP and DAP then increased significantly and maintained a plateau from 2 to 29 minutes. Similarly, Group TT showed a drop in MAP and DAP at 0.5 minutes and resumed baseline levels after vasopressin administration. Thereafter, the MAP and DAP elevated significantly from 3 to 7 minutes and the peak levels were higher than those of other groups. The DAP demonstrated no changes and the MAP demonstrated one occasional rise at 4 minutes in Group Placebo and at 7 minutes in Group LMA (figure 1,2).

The heart rates in Group Placebo were higher than in other groups, but there were no statistically significant differences between Group Control and TT, or Group Placebo and LMA. Groups Placebo, TT and LMA showed no persistent changes in heart rate after vasopressin administration. In Group LMAC, the heart rate decreased significantly at 2 minutes after drug delivery and maintained a lower level until 29 minutes (figure 3).

\section{Discussion}

Vasopressin administered via tracheal tube elevated the MAP and DAP in the experimental animals, a finding that is in line with previous research. (23-25) Nevertheless, vasopressin delivered from the LMA did not demonstrate any apparent hemodynamic changes, which limits the application of vasopressin administration directly from a LMA. The insignificant hemodynamic changes may be due to several factors. First, vasopressin injected from the upper end of a LMA sprays most of the 
drug on the mucosa of the larynx instead of the much larger surface of the bronchoalveolar system. The smaller surface limits the absorption of vasopressin and results in unobvious pharmacologic effects. Moreover, during forceful artificial ventilations after drug delivery, ventilating air and drugs might leak from the lower aperture of the LMA, which further impedes the distribution of vasopressin. $(6,26)$

The vasopressin in Group LMAC presented rapid, constant, and prolonged hemodynamic changes. The catheter aerosolized the injected solution into fine particles, which distributed the drugs to the bronchoalveolar system well and improved drug absorption. We conducted a manikin study for the feasibility of catheter placement through a LMA and found that placement of the catheter was fast, easy, and required minimal training. (27) Accordingly, vasopressin should be administered via a catheter passing through a LMA.

For the management of hemorrhagic shock, early large-volume fluid infusions have not proven to be effective due to the number of complications, including coagulopathy, multiorgan failure, promotion of additional blood loss, and hypothermia. $(28,29)$ Nevertheless, the delay in fluid resuscitation causes a shortage of requisite end-organ perfusion that can result in early mortality in severe trauma patients. $(11,12)$ Animal studies demonstrate that vasopressin increases blood pressure and prevents hypovolemic cardiac arrest from fatal hemorrhage. $(11,12)$ In addition, to improve perfusion pressure of vital organs, exogenous administration of vasopressin provides the added benefit of enhancing clot formation. (13) Therefore, it seems reasonable to consider vasopressin for treating hemorrhagic shock patients at the scene.

In patients with septic shock, the development of relative vasopressin deficiency provides the rationale for using vasopressin as an adjunct to catecholamine. $(14,15)$ Neto et al. conducted a systemic review and metaanalysis on the use of vasopressin and its analogue in critically ill patients. It was found that vasopressin decreased mortality in adult patients and in patients with septic shock. (16) Observational studies have shown some benefits involving early, low-dose vasopressin 
administration in patients with septic shock. $(14,17)$ Our experiment demonstrated that vasopressin administered via an aerosolized catheter through a LMA produces an obvious and constant pressor effect, which implies the possibility of a favorable influence on pre-hospital application of vasopressin.

The cardiopulmonary resuscitation guidelines for the American Heart Association have recommended intravenous vasopressin for the treatment of adult patients with cardiac arrest. (18) Various human and animal studies have also shown beneficial effects from the addition of vasopressin during resuscitation, including increased coronary perfusion pressure, cerebral oxygen delivery, the probability of restoring spontaneous circulation, and better neurologic outcome. (19-21) For cardiogenic shock patients, Jolly et al. found that vasopressin increased mean arterial pressure and displayed no adverse cardiac effect. (22) This evidence further supports the use of vasopressin in cardiopulmonary resuscitation, treatment of patients with cardiogenic shock, and cerebral hemorrhage. Furthermore, in our experiment, we found that only one dose of vasopressin, elevated arterial pressures for at least 27 minutes in Group LMAC. The effectiveness of a single dose of vasopressin could be sufficient for pre-hospital resuscitation and transportation in an urban area. $(30,31)$

We found that vasopressin significantly reduces heart rate, and the bradycardiac effect could prevent the progression of tachycardia induced by cardiomyopathy. (32) Diastolic dysfunction is a major predictor of mortality in patients with septic shock and reduction of the heart rate in patients with diastolic dysfunction can achieve better ventricular filling. (33) If preexisting bradycardia is found before vasopressin administration, pre-treatment with tracheal atropine can antagonize the effect. (34)

There were several limitations that influenced the application of the study results. First, the LMA is designed for humans and fits loosely in a pig larynx. Obvious air leaks were expected when artificial ventilations were conducted via LMA in the experimental pigs, which may 
compromise the absorption of vasopressin. In addition, as all of the pigs were healthy prior to the experiment, the pharmacodynamics in a healthy porcine model may differ from animals in cardiac arrest or shock states.

\section{Conclusion}

In this porcine model, vasopressin, administered via an aerosolized catheter and ventilated with a LMA, demonstrated a positive and prolonged pressor effect. The results suggest that an aerosolized catheter through a LMA may be a practical alternative route for vasopressin administration, to provide a rapid and constant drug effect.

Furthermore, the effective duration of LMA-administered vasopressin is long enough to cover the interval for pre-hospital resuscitation and transportation. Because placement of a LMA and catheter are fast and easy, we suggest conducting further studies for the utilization of vasopressin, aerosolized catheters, and LMAs in pre-hospital settings.

\section{References}

1. Smith RM, Conn AK. Prehospital care - scoop and run or stay and play? Injury 2009;40: suppl 4S:23-6.

2. Liberman M, Mulder D, Lavoie A, Denis R, Sampalis JS. Multicenter Canadian study of prehospital trauma care. Ann Surg 2003;237:153-60.

3. Bochicchio GV, Scalea TM. Is field intubation useful? Curr Opin Crit Care 2003;9:524-9.

4. Isenberg DL, Bissell R. Does advanced life support provide benefits to patients?: A literature review. Prehosp Disaster Med 2005;20:265-70.

5. Reades R, Studnek JR, Vandeventer S, Garrett J. Intraosseous versus intravenous vascular access during out-of-hospital cardiac arrest: a randomized controlled trial. Ann Emerg Med 2011;58:509-16.

6. Pollack CV Jr. The laryngeal mask airway: a comprehensive review for the Emergency Physician. J Emerg Med 2001;20:53-66.

7. Ostermayer DG, Gausche-Hill M. Supraglottic Airways: The History and 
Current State of Prehospital Airway Adjuncts. Prehosp Emerg Care 2013 Sep 12. [Epub ahead of print]

8. Chen KT, Lin HJ, Guo HR, Lin MT, Lin CC. Feasibility study of epinephrine administration via laryngeal mask airway using a porcine model. Resuscitation 2006;69:503-7.

9. Chen KT, Lin HJ, Jeng HW, Lin CC, Guo HR. The pharmacological effect of epinephrine administration via laryngeal mask airway in a porcine model of asphyxial cardiac arrest. Emerg Med J 2008;25:722-4.

10. Liao CK, Lin HJ, Foo NP, Lin CC, Guo HR, Chen KT. Epinephrine administration via a laryngeal mask airway: what is the optimal dose? Signa Vitae 2010;5:25-8.

11. Raedler C, Voelckel WG, Wenzel V, Krismer AC, Schmittinger CA, Herff $\mathrm{H}$, et al. Treatment of uncontrolled hemorrhagic shock after liver trauma: fatal effects of fluid resuscitation versus improved outcome after vasopressin. Anesth Analg 2004;98:1759-66.

12. Bayram B, Hocaoglu N, Atilla R, Kalkan S. Effects of terlipressin in a rat model of severe uncontrolled hemorrhage via liver injury. Am J Emerg Med 2012;30:1176-82.

13. Rezende-Neto JB, Rizoli SB, Andrade MV, Ribeiro DD, Lisboa TA, Camargos ER, et al. Permissive hypotension and desmopressin enhance clot formation. J Trauma 2010;68:42-50.

14. Holmes CL, Patel BM, Russell JA, Walley KR. Physiology of vasopressin relevant to management of septic shock. Chest 2001;120:989-1002.

15. Patel BM, Chittock DR, Russell JA, Walley KR. Beneficial effects of shortterm vasopressin infusion during severe septic shock. Anesthesiology 2002;96:576-82.

16. Serpa Neto A, Nassar AP Júnior, Cardoso SO, Manetta JA, Pereira VG, Espósito DC, et al. Vasopressin and terlipressin in adult vasodilatory shock: a systematic review and meta-analysis of nine randomized controlled trials. Crit Care 2012 Aug 14;16(4):R154.

17. Lange M, Ertmer C, Rehberg S, Morelli A, Köhler G, Kampmeier TG, et al. Effects of two different dosing regimens of terlipressin on organ functions in ovine endotoxemia. Inflamm Res 2011;60:429-37.

18. Neumar RW, Otto CW, Link MS, Kronick SL, Shuster M, Callaway CW, et al. Part 8: adult advanced cardiovascular life support: 2010 American 
Heart Association Guidelines for Cardiopulmonary Resuscitation and Emergency Cardiovascular Care. Circulation 2010;122(18 Suppl 3): S729-67.

19. Morris DC, Dereczyk BE, Grzybowski M, Martin GB, Rivers EP, Wortsman J, Amico JA. Vasopressin can increase coronary perfusion pressure during human cardiopulmonary resuscitation. Acad Emerg Med 1997;4:878-83.

20. Dudkiewicz M, Proctor KG. Tissue oxygenation during management of cerebral perfusion pressure with phenylephrine or vasopressin. Crit Care Med 2008;36:2641-50.

21. Krismer AC, Wenzel V, Stadlbauer KH, Mayr VD, Lienhart HG, Arntz HR, Lindner KH. Vasopressin during cardiopulmonary resuscitation: a progress report. Crit Care Med 2004;32(9 Suppl):S432-5.

22. Jolly S, Newton G, Horlick E, Seidelin PH, Ross HJ, Husain M, Dzavik V. Effect of vasopressin on hemodynamics in patients with refractory cardiogenic shock complicating acute myocardial infarction. Am J Cardiol 2005;96:1617-20.

23. Efrati O, Barak A, Ben-Abraham R, Modan-Moses D, Berkovitch M, Manisterski $Y$, et al. Should vasopressin replace adrenaline for endotracheal drug administration? Crit Care Med 2003;31:572-6.

24. Efrati O, Barak A, Ben-Abraham R, Weinbroum AA, Lotan D, Manistersky Y, et al. Hemodynamic effects of tracheal administration of vasopressin in dogs. Resuscitation 2001;50:227-32.

25. Lin PC, Lin HJ, Guo HR, Lin CC, Chen KT. A comparison of the hemodynamic effects of tracheal administration of vasopressin, adrenaline, and vasopressin plus adrenaline in a porcine model. J Acute Med 2012;2:103-6.

26. Keller C, Puhringer F, Brimacombe JR. Influence of cuff volume on oropharyngeal leak pressure and fibreoptic position with the laryngeal mask airway. Br J Anaesth 1998;81:186-7.

27. Liao CK, Lin HJ, Chen KT. An easy method to administer drugs into the trachea via the intubating laryngeal mask airway. Am J Emerg Med 2008 Mar;26(3):370-1.

28. Bickell WH, Wall MJ Jr, Pepe PE, Martin RR, Ginger VF, Allen MK, Mattox KL. Immediate versus delayed fluid resuscitation for hypotensive 
patients with penetrating torso injuries. N Engl J Med 1994;331:1105-9.

29. Revell M, Greaves I, Porter K. Endpoints for fluid resuscitation in hemorrhagic shock. J Trauma 2003;54(5 Suppl): S63-7.

30. Spaite DW, Bobrow BJ, Vadeboncoeur TF, Chikani V, Clark L, Mullins T, Sanders AB. Resuscitation 2008 Oct;79(1):61-6.

31. Spaite DW, Stiell IG, Bobrow BJ, de Boer M, Maloney J, Denninghoff K, et al. Ann Emerg Med 2009 Aug;54(2):248-55.

32. Richard C. Stress-related cardiomyopathies. Ann Intensive Care 2011;1:39.

33. Landesberg G, Gilon D, Meroz Y, Georgieva M, Levin PD, Goodman S, et al. Diastolic dysfunction and mortality in severe sepsis and septic shock. Eur Heart J 2012;33:895-903.

34. Efrati O, Modan-Moses D, Ben-Abraham R, Bibi H, Paret G. Atropine aborts bradycardic effect of endotracheally administered vasopressin. Med Sci Monit 2005;11: CR410-4.

Table 1. Biometric data.

\begin{tabular}{|c|c|c|c|c|}
\hline Group & Placebo & $\mathrm{TT}$ & LMA & LMAC \\
\hline $\mathrm{M} / \mathrm{F}$ & $2 / 4$ & $2 / 4$ & $1 / 5$ & $3 / 3$ \\
\hline $\begin{array}{l}\text { Weight (kg; average } \pm \\
\text { SD) }\end{array}$ & $\begin{array}{l}21.6 \pm \\
3.5\end{array}$ & $\begin{array}{l}22.1 \pm \\
3.4\end{array}$ & $\begin{array}{l}22.0 \pm \\
3.4\end{array}$ & $\begin{array}{l}22.5 \pm \\
2.2\end{array}$ \\
\hline
\end{tabular}

\section{Figure 1.}

In Group TT and Group LMAC, the MAP decreased significantly at 0.5 minutes and returned to baseline soon after, at 1 minute after drug administration. The MAP then increased significantly and maintained a plateau from 3 to 7 minutes in Group TT and from 2 to 29 minutes in Group LMAC. The peak level of MAP in Group TT was higher than in other groups. Furthermore, the MAP demonstrated one occasional rise at 4 minutes in Group Placebo and at 7 minutes in Group LMA.

LMA, laryngeal mask airway; LMAC, laryngeal mask airway and a 
catheter; MAP, mean arterial pressure; TT, tracheal tube.

**: The MAP is significantly higher than the baseline, $\mathrm{p}<0.05$

***: The MAP is significantly lower than the baseline, $\mathrm{p}<0.05$
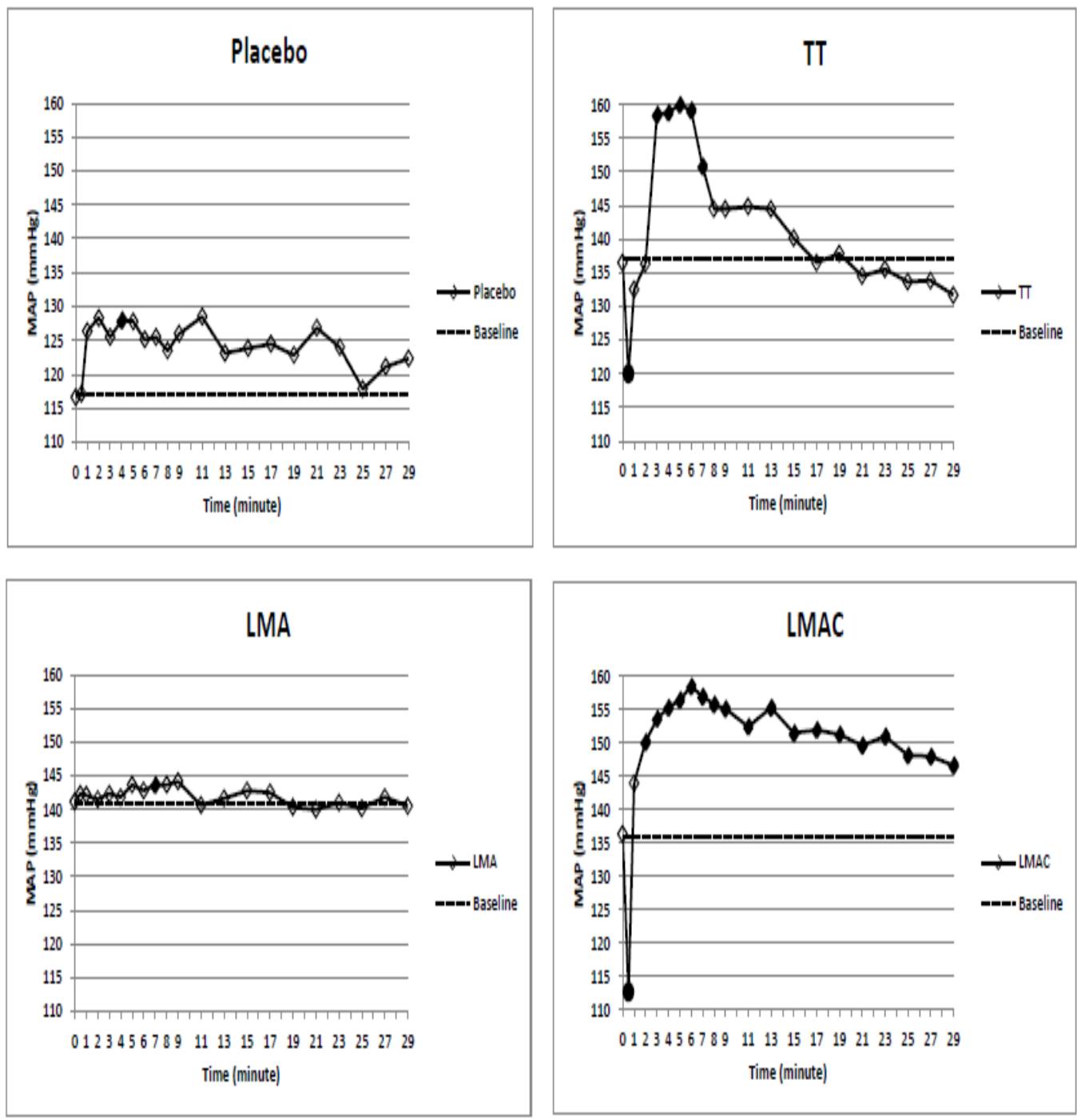

Figure 2.

In Group TT and Group LMAC, the DAP decreased significantly at 0.5 minutes and returned to baseline soon after, at 1 minute after drug administration. The DAP then increased significantly and maintained a plateau from 3 to 7 minutes in Group TT and from 2 to 29 minutes in Group LMAC. The peak level of DAP in Group TT was higher than in other groups. In Group Placebo and Group LMA, the DAP demonstrated 
no significant differences from the baseline.

DAP, diastolic arterial pressure; LMA, laryngeal mask airway; LMAC, laryngeal mask airway and a catheter; TT, tracheal tube.

**: The DAP is significantly higher than the baseline, $\mathrm{p}<0.05$

***: The DAP is significantly lower than the baseline, $\mathrm{p}<0.05$
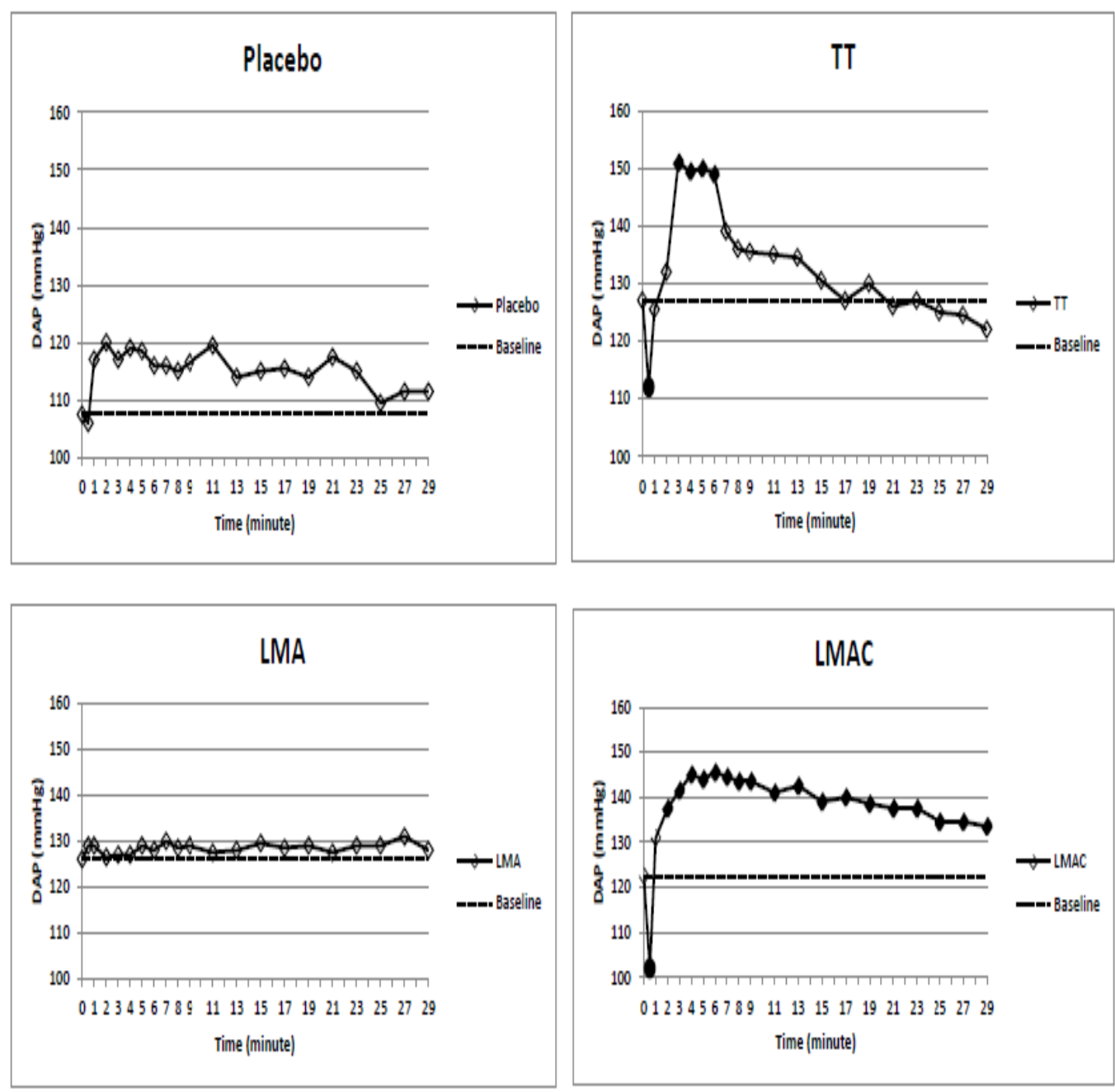

\section{Figure 3.}

The heart rate after drug administration was highest in Group Placebo and lowest in Group LMAC. In Groups Placebo, TT and LMA, the heart rate showed no constant changes after drug delivery. In Group LMAC, the heart rate decreased significantly at 2 minutes after vasopressin administration and the bradycardiac effect was prolonged. 
HR, heart rate; LMA, laryngeal mask airway; LMAC, laryngeal mask airway and a catheter; TT, tracheal tube.

**: The HR is significantly lower than the baseline, $\mathrm{p}<0.05$
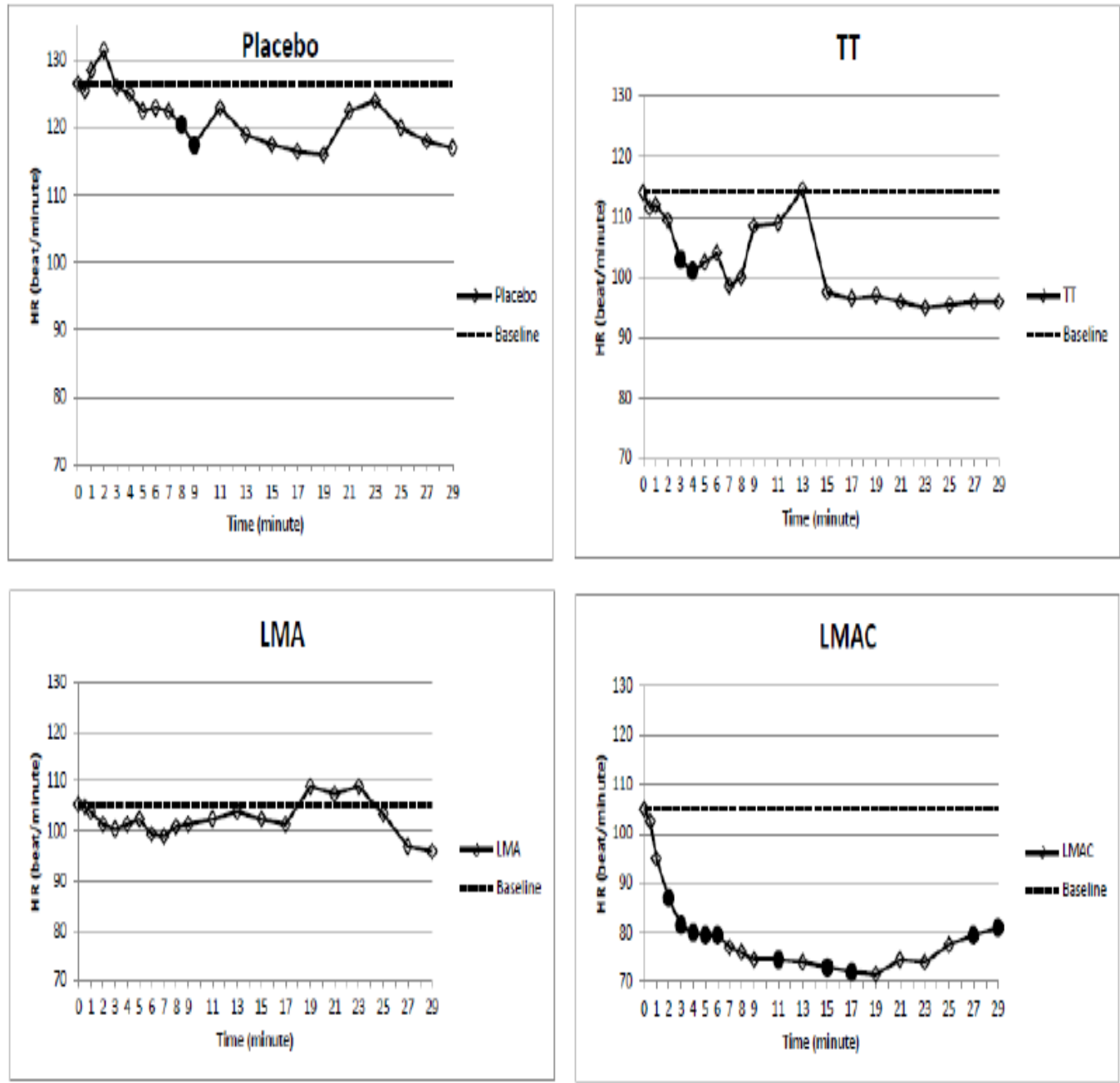

Yu-Ying Liao

Emergency Department, Chi-Mei Medical Center, Liouying, Tainan, Taiwan

Chien-Chin Hsu, Kuo-Tai Chen

Emergency Department, Chi-Mei Medical Center, Tainan, Taiwan

Chien-Chin Hsu

Department of Biotechnology, Southern Taiwan University of Science and Technology, Tainan, Taiwan

How-Ran Guo

Department of Environmental and Occupational Health, Medical College, National Cheng Kung University, Tainan, Taiwan

Kuo-Tai Chen

Emergency Department, Taipei Medical University, Taipei, Taiwan 
Address for reprints:

Kuo-Tai Chen

Emergency Department

Chi-Mei Medical Center

901 Chung-Hwa Road, Yung Kang

Tainan 710, Taiwan

Phone: 886-6-2812811 ext. 57196;

Fax: 886-6-2816161

E-mail: 890502@mail.chimei.org.tw

Article printed from Signa Vitae: http://www.signavitae.com

URL to article: http://www.signavitae.com/2016/o5/thefeasibility-of-vasopressin-administration-via-laryngeal-maskairway-using-a-porcine-model/

Copyright (C) 2015 Signa Vitae. All rights reserved. 